\title{
Derivation of Hubble's Law and the End of the Darks Elements
}

\author{
João Carlos Holland de Barcellos \\ Universidade de Sao Paulo, Sao Paulo, Brazil \\ Email: jocaxx@gmail.com
}

How to cite this paper: Barcellos, J.C.H. (2019) Derivation of Hubble's Law and the End of the Darks Elements Open Access Library Journal, 6: e5338.

https://doi.org/10.4236/oalib.1105338

Received: March 14, 2019

Accepted: April 7, 2019

Published: April 10, 2019

Copyright ( 2019 by author(s) and Open Access Library Inc.

This work is licensed under the Creative Commons Attribution International License (CC BY 4.0).

http://creativecommons.org/licenses/by/4.0/

\author{
Abstract \\ From the "Decreasing Universe" model, establishing the contraction of space \\ tissue when exposed to a gravitational field, we derive the Hubble Law and, \\ from there, we will explain the effects "Dark Energy" and "Dark Matter".

\section{Subject Areas} \\ Modern Physics, Theoretical Physics

\section{Keywords} \\ Hubble's Law, Dark Energy, Dark Matter, Decreasing Universe, Universe \\ Expansion
}

\section{Introduction}

We know from observation that galaxies seem to move away in an accelerated way. This observation was synthesized in the famous "Hubble Law" [1].

To explain this accelerated scape an entity was proposed that became known as "Dark Energy" [2].

Subsequently, it was also observed that stars orbit galaxies at a much higher velocity than calculated. As if there was much more matter inside the galaxies than the one observed.

To explain this phenomenon another entity was proposed that became known as "Dark Matter" [3].

However, despite many efforts, no other evidence of both "Dark Energy" and "Dark Matter" has been found.

So we are proposing a new explanation that replaces these two "dark" entities with another hypothesis: The "Decreasing Universe" Hypothesis [4].

By reducing the number of hypotheses-from two to one-we will be in 
agreement with the "Ocam Razor", beyond which, as we shall see, we can also derive the "Hubble Law".

In the "Decreasing Universe" model [4] it is established that the gravitational field causes a space contraction that can be detected by an observer who is not subjected to such field. In this way, all objects within this space are also contracted, especially instruments of these observers.

Particularly our planet is subjected, in a greater or lesser degree, to various fields: The gravitational field of the Earth itself, the Sun, the Moon, the distant galaxies, and on.

If, for example, we have a measuring instrument as a scaler with the length " $\mathrm{L}$ " (=1 meter), then, from the point of view of an observer who does not suffer gravitational influence, it will notice that this scaler, with the time, will decrease in size.

Of course, for observers subject to these there will be no change, since all space and everything that is immersed in it is contracting at the same time so that it will have no change in the measurements made by them.

For example, here on Earth, a table with $2 \mathrm{~m}$ length, after millions of years, will continue to measure $2 \mathrm{~m}$, as the table shrinks in the same proportion as the measuring scaler and, locally, no difference can be observed.

\section{Outer Space}

However, in the intergalactic space the gravitational field is practically null, and, therefore, this space does not suffer the same contraction that we here on Earth are undergoing.

Thus, in the absence of a considerable gravitational field in intergalactic space, the space between us and a distant galaxy will not contract in the same proportion as our own terrestrial space is contracting.

Consider, for example, the enormous time a photon, emitted by a distant galaxy, takes to reach us. In this long period of time, which may be billions of years from the emission of the photon until it reaches our planet, our space-and our scalers-will be reduced in size compared to the original size they had when this photon was emitted under the view of an observer who is not subject to such a gravitational field.

This reduction of our local space and the size of our "scalers" will cause us to distance ourselves to the star larger than it was at the time the photon was emitted (even if its actual distance did not change in that period).

\section{Defining Some Concepts}

Let's call of "Local Space" (="LS") the region of space that is subject to a non-negligible gravitational field and thus suffers spatial contraction.

Let's call of "Local Observer" (="LO") the observer who belongs to a "LS" and therefore subject-him and his instruments-the spatial contraction. For example, the planet Earth is an "LS" and we are "LO". 
Let's call of "Outer Space" (="OS") the region of space that is subject to a very weak and despicable gravitational field.

Let's call of "Sidereal Observer" (="SO") those observers located in this spatial region. For example, observers in the intergalactic region would be an "OS".

To clarify ideas, we may think that observers in the "OS" (="SO") play a role similar to an observer in an inertial frame [5] as opposed to observers located in the "LS" that would play an analogous role to observers in a non-inertial referential.

\subsection{Exemplifying the Concepts}

Consider, for example, at an arbitrary initial instant any " $t_{0}$ " in the "LS", a scaler of length " $L_{0}$ " that a "LO" uses to make its measurements.

Suppose that at this moment " $t_{0}$ " an "SO", in intergalactic space, take this measure of this same " $L_{0}$ " scaler as the standard measure for your own measurements.

Then, at time " $t_{0}$ ", both observers (" $L O$ " and " $S O$ ") will consider the pattern " $L_{0}$ " of the same size.

However, the "LS" will continue to contract in relation to the "OS". The "LO" will not notice the variation of the scaler " $L_{0}$ " because both its measuring scaler and everything in his "LS" decrease in the same proportion. However, the "SO", after a time " $t$ " (" $t$ " $>$ " $t_{0}^{\prime \prime)}$ will see the scaler of the "LO" decrease to a smaller size " $L$ ".

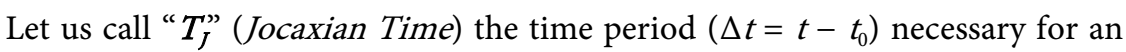
"SO" see the space (and the scaler) of the OL contracting at half size it had at time $t_{0}$. That is, to shrink itself to a size $L=L_{0} / 2$ at time $t_{0}+T_{\dot{f}}$.

Before we go on let's make some simplifications.

\subsection{Some Simplifications}

Before we continue, we will consider that:

- If the gravitational field is constant, the time required for the "LS" (and all that is contained in it), to contract to half its size, called TJ, measured by an "SO", will also be constant.

We will also consider that the galaxies, from the point of view of an "SO" are not necessarily rapidly moving away from each other. For simplicity we will calculate the effects of "Dark Energy" and "Dark Matter" only as a result of our gravitational contraction, keeping constant its distances (from the point of view of an "SO").

Also we will not consider the effect of time dilation [6] due to the gravitational force in the "LS" in relation to the "OS".

\section{Local Space Contraction Formula ("LS")}

We can mathematically translate the concepts we saw above into the following formula: 


$$
L(t)=\frac{L_{0}}{2^{\Delta t / T_{j}}}
$$

(Formula of space contraction from the point of view of an "SO") where:

$L(t)=$ Measure of $L_{0}$ in the "LS" by a "Sidereal Observer"

$t_{0}=$ Initial time (arbitrary)

$L_{0}=$ Length measured in $t=t_{0}$

$T_{j}=$ Jocaxian Time

$\Delta t=t-t_{0}$

Note that for an "LO", $L=L_{0}$ (always!), that is, the size of the scaler does not change with time in the "LS".

At each " $T_{J}$ ” period of time, our space (and our scales) are contracted in half (from the point of view of an "SO").

If we define:

$$
F_{j}(\Delta t)=2^{\Delta t / T_{j}}
$$

(Jocaxian Factor).

We can rewrite (E1):

$$
L=L_{0} / F_{j}(\Delta t)
$$

We can also rewrite the same Jocaxian Factor $\left(F_{j}\right)$ in a more friendly way:

$$
F_{j}(\Delta t)=\mathrm{e}^{\left(\ln (2) * \frac{\Delta t}{T_{j}}\right)}
$$

\section{4. "Dark Energy" Effect}

Of course, if intergalactic space does not contract, and if our "scale of measurement" decrease in size, then this intergalactic space should seem to us larger, in the same proportion as our "scale of measurement" contract itself.

If, for example, at $t=t_{0}$, we measure the distance to a galaxy " $\mathrm{X}$ " with our

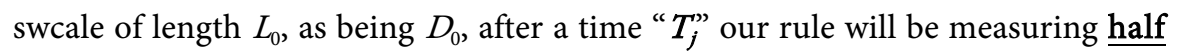
of its initial size $L_{0}$, and therefore, when we ("LO") measure the distance to that galaxy, we will measure it as being $D=2 * D_{0}$

We should note that a measure within our "LS" sizes do not change, as everything decreases along with our scaler and rulers, but the "OS" does not contract like our "LS". So we will have this illusion that the galaxy " $\mathrm{X}$ " is moving away from us. This is what we can call the "Dark Energy Effect".

\section{Apparent Distance Formula}

The measured distance is inversely proportional to the length of the measurement pattern. We can synthesize this idea mathematically with the following formula (see the Appendix A):

$$
D(\Delta t)=D_{0} * F_{j}(\Delta t)
$$

(Distance Formula with the Jocaxian Factor). 
where:

" $t_{0}$ " is the time at which the photon was emitted by the galaxy.

" $t$ " is the time at which Earth received this photon.

" $D_{0}$ " is the distance we would measure, from Earth to galaxy at time " $t_{0}$ ".

" $D(\Delta t)$ " is the distance we measured from Earth to a galaxy after " $\Delta t$ " time.

“ $\Delta t$ " $=t-t_{0}$ Time period.

As $F_{j}(\Delta t)$ grows exponentially with time (E3) the Earth's distance from the galaxy will also appear to increase exponentially with time.

\section{Hubble's Law}

With the formula of the apparent distance (E4) we can calculate the apparent distance speed:

$$
\begin{gathered}
V=\partial[D(\Delta t)] / \partial t \\
V=\partial\left[D_{0} * \mathrm{e}^{\left(\ln (2) * \frac{\Delta t}{T_{j}}\right)}\right] / \partial t \\
V=\left(\frac{\ln (2)}{T_{j}}\right) * D(\Delta t)
\end{gathered}
$$

(Distant galaxies apparent distance speed formula.)

But Hubble's law is exactly like this:

$$
V=H_{0} * D
$$

(Hubble's Law)

where ( $H_{0}=$ Hubble's Constant and $D$ is the distance from the galaxy)

As E6 $=\mathrm{E} 7$, now we can determine $T_{\dot{j}}$

$$
T_{j}=\frac{\ln (2)}{H_{0}}
$$

Substituting (E8) into (E3) we will have:

$$
F_{j}(\Delta t)=\mathrm{e}^{H_{0} * \Delta t}
$$

(Jocaxian Factor in terms of the Hubble constant)

What provides us:

$$
D(\Delta t)=D_{0} * \mathrm{e}^{H_{0} * \Delta t}
$$

(Apparent distance formula in terms of the Hubble constant)

If we want to calculate the real distance from Earth to the galaxy, using the measurements that our scales had at the time the photon was emitted (at $t=t_{0}$ ) then:

$$
\Delta t=\frac{D_{0}}{c}
$$

(Time for a photon emitted from the galaxy to reach us, where $c=$ speed of light) 
From (E11) and (E10) we will have:

$$
D=D_{0} * \mathrm{e}^{D_{0} * H_{0} / c}
$$

(Apparent distance of a galaxy depending on the actual distance)

\section{Some Values}

As $H_{0}=2.2 \mathrm{e}-18 \mathrm{~s}^{-1}$, we can replace it in (E8) and find $T_{j}$

$$
T_{j}=3.15 \mathrm{E} 17 \mathrm{~s}=10 \text { billion years }
$$

That is, the Jocaxian Time, the time necessary for our space to contract in half, is 10 billion years.

We can now find the contraction rate of our space for every billion years:

$$
(T x)^{10}=2 \Rightarrow T x=\mathrm{e}^{\ln (2) / 10}=7 \%
$$

That means:

For every 1 billion years our space (and our scalers) are contracted 7\% of their original size.

It is interesting to note that this value (7\%) corresponds exactly to the contraction rate calculated from the "Redshift" of the Galaxy NGC3034 [7].

Currently the apparent distance of the "NGC3034" is about 11E6 light years, (or 1E23 meters).

Applying (E12) to the galaxy NGC3034 and knowing that $H_{0} / c=8 \mathrm{E}-27 \mathrm{~m}^{-1}$ we will have the following equation for the distance to the galaxy NGC3034:

$$
10^{23}=D_{0} * \exp \left(D_{0} * 8 * 10^{-27}\right)
$$

(Equation of the real distance of the Galaxy NGC3034)

Using a solver [8] we will obtain for the real distance:

$D_{0}=9 \mathrm{E} 22$ that is, this galaxy is about $10 \%$ closer to Earth than it appears to be.

\section{Dark Matter}

Dark Matter [3] can also be observed being an effect of our spatial contraction.

As nomenclature, we will suppress the subscripts "obs" of the measurements observed here from Earth. So we'll simplify:

$V_{\text {obs }}=V$; (Rotation Speed Observed)

$D_{\text {obs }}=D ;$; (Distance Observed)

$R_{\mathrm{obs}}=R$; (Radius Observed)

$W_{\text {obs }}=W ;$ (Angular Speed Observed)

$M_{\mathrm{obs}}=M$; (Mass Observed)

Considering the illustration:

Suppose that from Earth we now observe a star circling the periphery of a galaxy that is at an observed (apparent) distance " $D$ " from our planet. According to Figure 1, the observed " $R$ " radius of the galaxy will be proportional to this distance: 


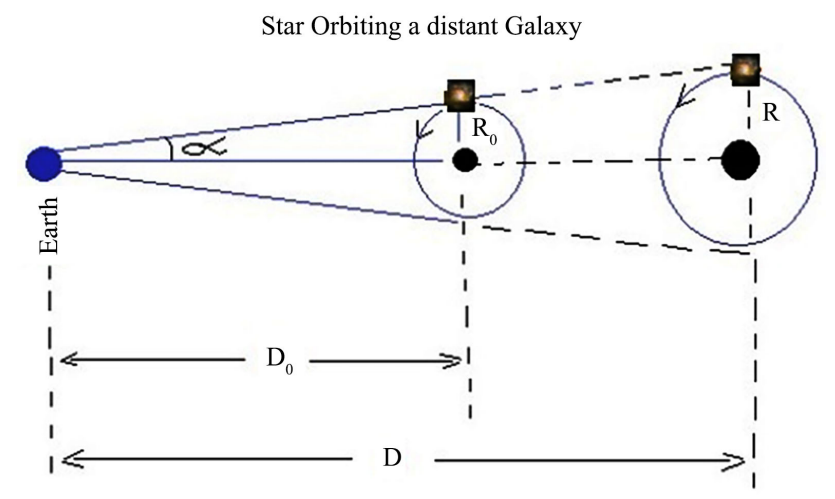

Figure 1. Show how we observe a star rotating a distant galaxy.

$$
R=\operatorname{sen}(\alpha) * D
$$

(Orbit radius as a function of observed distance and angle)

As we saw earlier, at the time the photon was emitted, the real distance would be $D_{0}$, so:

$$
R_{0}=\operatorname{sen}(\alpha) * D_{0}
$$

(Real orbit radius as a function of actual distance and angle)

From (E9) and (E11) We can define the Jocaxian Factor of the Galaxy:

$$
F_{j}=\exp \left(D_{0} * \frac{H_{0}}{c}\right)
$$

(Jocaxian Factor of the Galaxy)

So, we will have:

$$
R_{0}=\frac{R}{F_{j}}
$$

(Real Radius as a function of the Jocaxian factor of the Galaxy)

If $M$ is the observed mass of the galaxy where the star orbits, and $V$ is its tangential velocity observed from the Earth, and $G$ is the Gravitational constant of the Galaxy, we will have [9]:

$$
V^{2}=M * \frac{G}{R}
$$

(Equation of Velocity as a function of mass and radius)

In terms of the angular velocity, we have:

$$
V^{2}=W^{2} * R^{2}
$$

(Equation of Velocity as a function of angular velocity and radius)

From (E19) and (E20) we derive:

$$
W^{2}=\frac{M G}{R^{3}}
$$

(Equation of the angular velocity as a function of Mass and Radius) From (E21), at $t=t_{0}$, we have: 


$$
W_{0}^{2}=\frac{M_{0} G}{R_{0}^{3}}
$$

(Equation of the angular movement in function of the Real Radius and Real Mass)

The angular velocity does not change with the observed distance, since the time interval between two emitted photons is the same interval when they arrive to Earth. So:

$$
W=W_{0}
$$

(The angular speed is the same for an " $L O$ " as for a "SO")

From (E20), (E22), (E23) we find:

$$
V^{2}=\left(M_{0} G / R_{0}^{3}\right) * R^{2}
$$

Using (E18):

$$
V^{2}=\left(M_{0} * F_{j}^{3}\right) * G / R
$$

(Equation of the tangential velocity as a function of the Barium Mass and Apparent Radius)

Comparing (E25) with (E19) we conclude that:

$$
M=M_{0} * F_{j}^{3}
$$

(Apparent mass as a function of actual mass)

From Earth we observe a mass $\mathrm{M}$ for the galaxy larger than the mass at $t=t_{0}$.

Then the effect "Dark matter" will be the difference of $M$ with the real mass $M_{0}$ :

$$
\text { Dark Matter }=M-M_{0}=M_{0} *\left(\exp \left(3 * D_{0} * \frac{H_{0}}{c}\right)-1\right)
$$

(Dark matter equation as a function of the Hubble constant and the actual distance)

\section{Conclusions}

If we adopt the "Decreasing Universe" where the gravitational field shrinks the space in which it crosses, we find that the accelerated separation of galaxies, often explained by the so-called "Dark Energy" is a kind of "illusion" resulting from this space contraction and, therefore, unnecessary.

The "Dark Matter", on the other hand, can also be explained by the same effect of the gravitational contraction of our space, since the radius of the galaxies is observed as greater than it really is; consequently, the speed of translation of a star is seen as above-expected with the observed baryonic mass, providing the false impression that there is an extra, invisible matter responsible for the effect.

\section{Conflicts of Interest}

The author declares no conflicts of interest regarding the publication of this paper. 


\section{References}

[1] Hubble's Law. https://en.wikipedia.org/wiki/Hubble\%27s_law

[2] Dark Energy. https://en.wikipedia.org/wiki/Dark_energy

[3] Dark Matter. https://pt.wikipedia.org/wiki/Dark_Matter

[4] The Gravitational Field and the Dark Energy. https://ijrdo.org/index.php/as/article/view/1311

[5] Inertial Frame of Reference. https://en.wikipedia.org/wiki/Inertial_frame_of_reference

[6] Gravitational Time Dilation. https://en.wikipedia.org/wiki/Gravitational_time_dilation

[7] The Equivalence Principle and the End of the Dark Energy. http://www.ijera.com/papers/Vol7_issue1/Part-1/C0701011314.pdf

[8] Solver for Equations. https://www.mathway.com/Algebra

[9] Orbital Speed. https://en.wikipedia.org/wiki/Orbital_speed 


\section{Appendix A}

\section{Derivation of the Distance Formula from the Local Contraction Formula.}

At $t=t_{0}$ we will take as the measurement standard for both observers the measure " $L_{0}$ ", for example, $L_{0}=1$ meter. Thus, all distances will be taken as a number that multiplies the $L_{0}$ pattern;

Then both observers, sidereal and terrestrial, measure the same distance to a given galaxy:

$$
\text { Distance }=D_{0} * L_{0}
$$

( $D_{0}$ is the distance that is measured to the galaxy by taking the measurement pattern $L_{0}$ )

After a time $t\left(>t_{0}\right)$, from the point of view of a sidereal observer, the terrestrial space shrank, and the rule tablet $L_{0}$ decreased to $L$ according to E1:

$$
L=\frac{L_{0}}{F_{j}(\Delta t)}
$$

(Spatial contraction formula (E2))

As in our hypothesis, from the point of view of the Sidereal Observer, the galaxy does not move away, the distance covered must be the same, that is:

$$
\text { Distance }=D * L
$$

( $D$ is the measured Distance to the galaxy according to the L pattern, by the Terrestrial observer)

We must keep in mind that for the local terrestrial observer, $L=L_{0}$, since he does not perceive his own contraction).

From (A1) and (A2) we have to:

$$
D_{0} * L_{0}=D * L
$$

(From the point of view of the sidereal observer, the distance is not altered)

Using (E1), we have finally:

$$
D=D_{0} * F_{j}(\Delta t)
$$

(Measured distance to the galaxy according to the terrestrial observer, as a function of time) 